\title{
Deaminations for diversification
}

J. Am. Chem. Soc. http://doi.org/cz27 (2019)
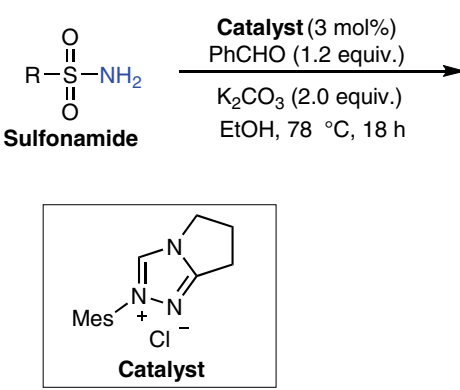

People working in drug discovery and development will be well aware of how often the sulfonamide group pops up in drug products. This includes the sulfa drugs very early antibiotics - and now a range of drugs with a wide variety of therapeutic uses. This functional group is typically viewed as a target to be installed in the final product, and not a functional handle for further manipulation.

Now chemists at Merck (Rahway) have developed a method to turn primary sulfonamides into something new, by removing or replacing either the $-\mathrm{NH}_{2}$ or the entire $-\mathrm{SO}_{2} \mathrm{NH}_{2}$ group. Condensing the sulfonamide with benzaldehyde in the presence of base and an $\mathrm{N}$-heterocyclic carbine catalyst led to remarkably high conversions to sulfonate, even in the case of sterically hindered starting materials (very little product was observed in the absence of catalyst). Common functional groups including carboxylic acids and anilines were compatible with the deamination procedure, showing the potential for late-stage diversification.

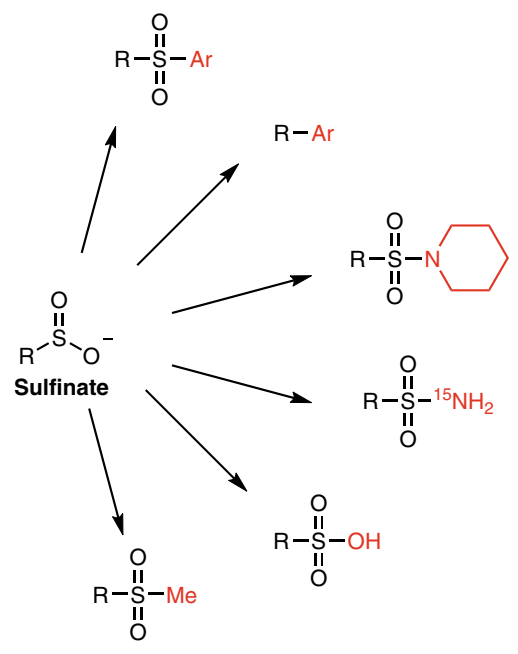

Also of note is the fact that all reagents used - including the NHC catalyst - are commercially available, and that the reaction can be run at high concentrations under mild conditions.

After showing that deamination could be achieved, the next step was to see what the resulting sulfinate could be converted to. Here the authors were not short of options; simple electrophiles such as methyl iodide could react to produce sulfones, and under oxidative conditions other amines (including ${ }^{15} \mathrm{~N}$-labelled reagents) could be added in place of the original primary amine group. The sulfonates could also take part in palladium cross-coupling reactions, either with retention of the $\mathrm{SO}_{2}$ group to form aryl sulfones, or with loss of $\mathrm{SO}_{2}$ to form biaryls. Overall, this work shows the untapped potential for using sulfonamides as functional handles.

\section{Enda Bergin}

Published online: 13 February 2019

https://doi.org/10.1038/s41929-019-0239-1 